\title{
Eosinophilic ulcer of the tongue - Case report
}

\author{
Dario Didona ${ }^{1}$ \\ Michele Donati ${ }^{3}$ \\ Stefano Calvieri ${ }^{2}$
}

\author{
Giovanni Paolino ${ }^{2}$ \\ Biagio Didona $^{1}$
}

DOI: http:/ / dx.doi.org/10.1590/abd1806-4841.20153600

\begin{abstract}
A bstract: Eosinophilic ulcer of the oral mucosa is a rare, self-limiting, chronic and benign lesion of unknown pathogenesis that affects the oral mucosa. We present the case of a 65 year-old Caucasian female with a fivemonth history of a painful ulcer on the lateral side of her tongue. The ulcer was not adhered to the underlying structures and there was no evidence of regional lymph node involvement. Laboratory examinations and X-rays revealed no abnormalities. Topical treatments had been performed without any improvement. Histopathological examination showed an ulcerated surface and mixed inflammatory infiltrate with several eosinophils extending into the mucosa and submucosa. No cellular atypia was observed. Based on the patient's history and mucosal biopsy, a final diagnosis of eosinophilic ulcer of the oral mucosa was made.
\end{abstract}

Keywords: Eosinophilic granuloma; Granuloma; Ulcer

\section{INTRODUCTION}

Eosinophilic ulcer of the oral mucosa (EUOM) is a rare self-limiting chronic benign lesion of the oral mucosa with unknown pathogenesis. ${ }^{1}$

Although the pathogenesis of EUOM is uncertain, an important role has been attributed to trauma caused by accidental bite, sharp teeth or hard food. ${ }^{2}$

Immunohistochemical studies have shown the expression of CD3, CD4, CD8, CD20, CD43, cytotoxic granules in the cellular infiltrate and the expression of CD30, vimentin and CD68 on the larger cells. ${ }^{1}$ The main differential diagnoses include Langerhans cells histiocytosis, Kimura's disease, angiolymphoid hyperplasia with eosinophilia, atypical histiocytic granuloma and CD30 lymphoproliferative disorder of the oral mucosa. ${ }^{1}$ As the condition is perhaps unknown to many dermatologists and due to the confusion in the literature with eosinophilic granuloma, we think the following case report deserves presentation.

\section{CASE REPORT}

A 65 year-old Caucasian female sought our Department with a five-month history of an approximately $2 \mathrm{~cm}$ painful ulcer on the tongue, adjacent to sharp teeth (Figure 1). The condition started as a slightly red lesion with mild induration, which rapidly underwent ulceration. The ulcer was not fixed to the underlying structures and there was no evidence of regional lymph node involvement. There was no history of previous dental treatment. The patient was a non-smoker and had no history of alcoholism. Laboratory examinations revealed no abnormalities, and X-rays revealed no affection to the bones of lower or upper teeth. The personal medical history was positive only for hypertension; skin diseases were not reported. The patient had previously undergone topical treatment with hyaluronic acid $0.6 \%$ and salicylic acid $0.5 \%$, without any improvement. 
Differential diagnoses included squamous cell carcinoma, non-Hodgkin's lymphoma, traumatic granuloma and EUOM. Regarding diagnosis, due to the clinical aspect of the mucosal lesion and lack of improvement with topical drugs, an incisional biopsy was performed. Histopathological examination showed an ulcerated surface with a significant number of eosinophils, extending into the mucosa and submucosa (Figure 2). No cellular atypias were observed.

Based on the patient's history and mucosal biopsy, a final diagnosis of EUOM was made. After 3 months of follow-up, the mucosal lesion improved without medical treatments and at 12-months follow-up, there was no recurrence.

\section{DISAUSSION}

EUOM is a rare benign entity. It was first described clinically by Riga in 1881 and histologically by Fede in $1890 .^{3}$

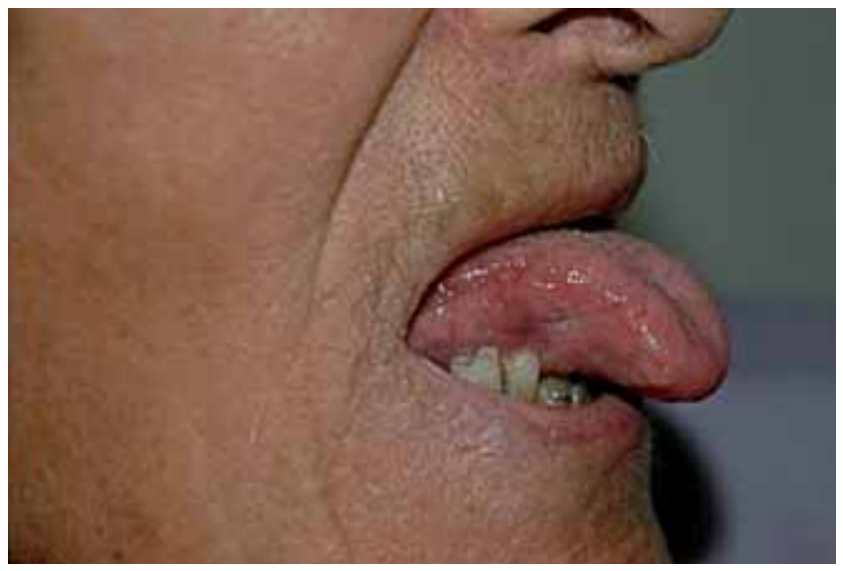

Figure 1: Ulcer on the right lateral border of the tongue, adjacent to two sharp teeth

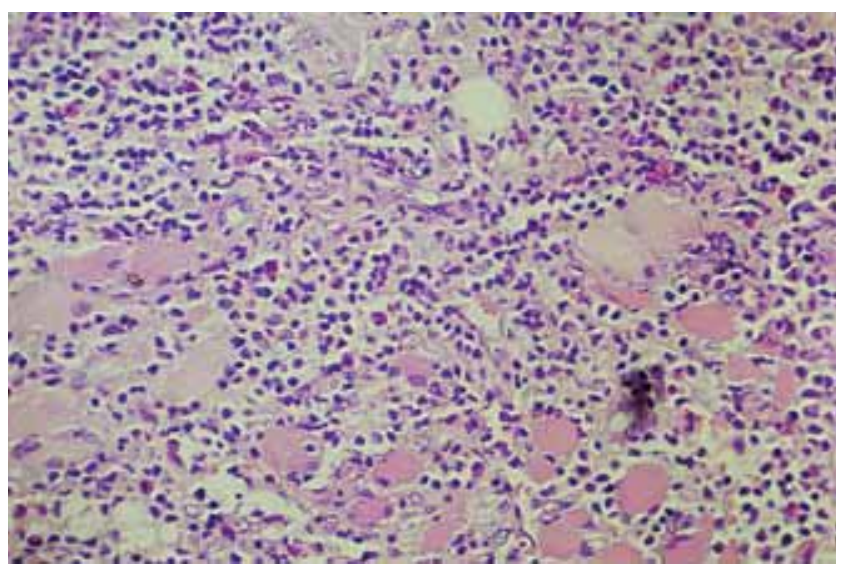

FIGURE 2: Submucosal infiltrate with many eosinophils (H\&E, X 400)
The elementary EUOM lesion is often a solitary ulcer, but Burges reported a case of multiple eosinophilic ulcers on the tongue of a boy affected by Riley-Day syndrome, a rare congenital insensitivity to pain. ${ }^{3}$

EUOM is described typically in early childhood or during the fifth decade of life, with a slightly higher prevalence in females. ${ }^{4}$ The clinical features of EUOM lead to several differential diagnoses, including mainly infectious diseases, malignancies and autoimmune diseases. ${ }^{5}$ However, since there is no specific hallmark for the disease, the diagnosis of EUOM is made by exclusion.

Although considered a rare entity, there are two large EUOM case series in the literature, suggesting that this oral disease could be more frequent than observed. ${ }^{2,4}$

More than half of the lesions occur on the tongue; other sites involved are lips, oral mucosa, gingiva, palate and the floor of the mouth. ${ }^{6}$ Pain or tenderness may be present in approximately $30 \%$ of cases. Most lesions are round ulcers with elevated and indurated borders; sometimes EUOM appears as a nodular-ulcerated lesion or simply as an induration; the last one can represent an early phase in the development of the typical ulcer. ${ }^{6}$

To date, the etiology of this disease remains unknown, although mechanical trauma (accidental bites, repeated thrusting against sharp or misplaced teeth), which coexists in more than half of the reported cases, has been considered an important trigger. ${ }^{7}$

Typically, histopathologic examination shows an inflammatory infiltrate with many eosinophils. Varying numbers of T-lymphocytes are present; T-cell specific antigen-presenting cells are more numerous than no antigen-presenting ones, suggesting that cell-mediated immunity may play an important role in the pathogenesis of EUOM. ${ }^{4}$ Currently it is not clear whether eosinophils and mast cells play an essential pathogenic role or are recruited by T-lymphocyte activation. The infiltrate can also contain a varying number of mast cells and mitotically active, large and atypical mononuclear cells, which have a myofibroblastic or histiocytic origin. ${ }^{4,8} \mathrm{Simin}$ et al. suggest a correlation between this pattern and other oral diseases with similar clinical and histopathological characteristics, such as angiolymphoid hyperplasia with eosinophilia, atypical histiocytic granuloma and Kimura's disease; other authors reported that infiltrating T-lymphocytes express CD30 antigen. ${ }^{1,7}$ On this topic, Alobeid et al. suggest that some cases of EUOM previously reported might be the oral manifestation of CD30 primary cutaneous lymphoproliferative disorders. ${ }^{9}$

An incisional biopsy is often required for definitive diagnosis when no evidence of spontaneous 
Chart 1: Therapeutic approach to EUOM

Wait-andsee approach

Surgical excision

Topical/ Intralesional/ Systemic

corticosteroids

Curettage

Antibiotics

Cryosurgery

healing is observed at 1-month follow up. Usually spontaneous healing occurs within 30 days, but it may require up to 8 months. Our case showed no recurrence, as normally reported in EUOM. ${ }^{5}$ Multiple lesions, either simultaneous or not, are described in the literature. ${ }^{5}$

\section{REFERENCES}

1. Bortoluzzi MC, Passador-Santos F, Capella DL, Manfro G, Nodari RJ Jr, Presta AA. Eosinophilic ulcer of oral mucosa: a case report. Ann Stomatol (Roma). 2012;3:11-3.

2. Elzay RP. Traumatic ulcerative granuloma with stromal eosinophilia (Riga-Fede's disease and traumatic eosinophilic granuloma). Oral Surg Oral Med Oral Pathol. 1983;55:497-506.

3. Eichenfield LF, Honig PJ, Nelson L. Traumatic granuloma of the tongue (Riga-Fede disease): association with familial dysautonomia. J Pediatr. 1990;116:742-4.

4. el-Mofty SK, Swanson PE, Wick MR, Miller AS. Eosinophilic ulcer of the oral mucosa. Report of 38 new cases with immunohistochemical observations. Oral Surg Oral Med Oral Pathol. 1993;75:716-22.

5. Mezei MM, Tron VA, Stewart WD, Rivers JK. Eosinophilic ulcer of the oral mucosa. J Am Acad Dermatol. 1995;33:734-40.

6. Vélez A, Alamillos FJ, Dean A, Rodas J, Acosta A. Eosinophilic ulcer of the oral mucosa: report of a recurrent case on the tongue. Clin Exp Dermatol. 1997;22:154-6.

7. Ada S, Seckin D, Tarhan E, Buyuklu F, Cakmak 0, Arikan U. Eosinophilic ulcer of the tongue. Australas J Dermatol. 2007;48:248-50.

8. Regezi JA, Zarbo RJ, Daniels TE, Greenspan JS. Oral traumatic granuloma. Characterization of the cellular infiltrate. Oral Surg Oral Med Oral Pathol. 1993;75:723-7.

9. Alobeid B, Pan LX, Milligan L, Budel L, Frizzera G. Eosinophilrich CD30+ lymphoproliferative disorder of the oral mucosa. A form of 'traumatic eosinophilic granuloma'. Am J Clin Pathol. 2004;121:43-50.

10. Gagari E, Stathopoulos P, Katsambas A, Avgerinou G. Traumatic ulcerative granuloma with stromal eosinophilia: a lesion with alarming histopathologic presentation and benign clinical course. Am J Dermatopathol. 2011;33:192-4.
Similarly to the experience of other authors, our case had a rapid improvement on the ulcer after the incisional biopsy. On this point, Eleni et al. consider that such behavior may be indicative of a healing response reactivation after the surgical intervention, but the reason for this improvement is to date unknown. ${ }^{10}$

In case of a persistent and rapidly growing lesion, surgical excision is the most used treatment procedure, among several different proposed therapies (Chart 1). Our case did not require surgical excision because it healed in less than 3 months. Although EUOM is a benign affection, monitoring is mandatory in all cases, as low-grade lymphoma may show a very similar clinical presentation in oral mucosa. ${ }^{10}$

How to cite this article: Didona D, Paolino G, Donati M, Didona B, Calvieri S. Eosinophilic ulcer of the tongue - Case report. An Bras Dermatol. 2015;90 (3 Suppl 1): S88-90. 